\title{
1er janvier 2012: la révolution n’a pas eu lieu
}

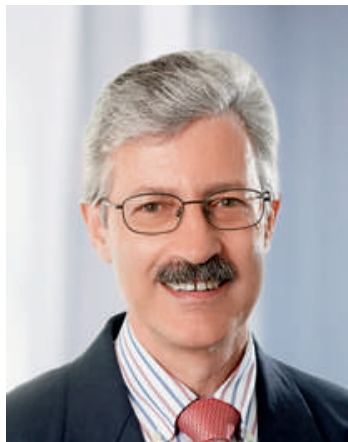

Depuis maintenant 5 mois, la nouvelle structure tarifaire SwissDRG est en vigueur dans toute la Suisse. Le changement n'a pas été perçu de la même manière à tous les échelons. Les patients sont ceux qui ont remarqué le moins de variation dans leur vie quotidienne. En effet, les processus de traitement n'ont pas subi de transformation d'un jour à l'autre et les critères d'efficacité sont restés naturellement prioritaires pour chacun d'entre nous. Au niveau administratif, des adaptations plus substantielles ont dû être

\section{La phase la plus difficile de la transition a été celle des négociations des taux de base.}

faites, tout manquement dans la représentation exacte des prestations effectuées étant immédiatement sanctionné par une pénalité financière. La phase la plus difficile de la transition a été celle des négociations des taux de base: si une solution satisfaisante a pu être trouvée dans certains cantons, dans d'autres en revanche, les partenaires tarifaires ne sont pas parvenus à s'entendre et des taux provisoires ont été fixés.
Malgré certaines manœuvres dilatoires, l'esprit de concurrence voulu par le législateur fédéral commence à être perceptible. Des collaborations sont en cours à l'échelle supra-cantonale, certaines concentrations hospitalières sont en voie de réalisation, trouvant un écho favorable auprès des patients qui privilégient la qualité et font preuve d'une compliance remarquable face aux changements. Plusieurs hôpitaux universitaires sont à l'origine d'une campagne visant à leur attirer des cas, contestant aux grands hôpitaux cantonaux la légitimité de toute une série de prestations lourdes. Il est bon que le problème de la masse critique soit abordé mais il devra être résolu sur la base de données scientifiques objectives.

La nouvelle structure tarifaire est évolutive et un rythme d'adaptation annuel a été convenu. Ainsi, le Conseil d'administration de SwissDRG SA a déjà entériné la version 2.0 qui sera appliquée dès le 1.1.2013. Celle-ci n'a pas permis de progresser significativement dans la représentation de l'activité hospitalière réelle, car il manque toujours un nombre important de rétributions additionnelles. Une discussion de fond aura prochainement lieu entre partenaires pour définir le degré de finesse que nous voulons atteindre avec une structure tarifaire qui demeure tout de même forfaitaire par définition.

Cela fait longtemps que la plupart des hôpitaux se sont préparés au nouveau financement hospitalier. L'informatisation se poursuit, la saisie des prestations s'améliore. La coordination des besoins administratifs, statistiques, épidémiologiques, comptables et de formation devient réalité. De nombreuses démarches se développent dans le domaine de la qualité. Après quelques soubresauts, bien des cantons reconnaissent maintenant une certaine autonomie de gestion aux hôpitaux. Toutes ces évolutions créent une dynamique dans laquelle le corps médical doit se positionner, de manière cri-

\section{Les évolutions autour de SwissDRG créent une dynamique dans laquelle le corps médical doit se positionner, de manière critique et en toute connaissance de cause, afin de conserver notre liberté thérapeutique intacte.}

Dans sa version 1.0, SwissDRG n'est pas encore très différencié. En particulier, les traitements spécialisés effectués dans les hôpitaux universitaires et les grands hôpitaux centraux ne sont pas encore pris en compte à leur juste valeur. Les actionnaires de SwissDRG SA sont conscients de cet état de fait et estiment indispensable, dans une phase initiale, de corriger cela par des taux de base distincts en fonction de la catégorie hospitalière. Certains ne l'ont cependant pas compris et pensent pouvoir comparer d'emblée tous les établissements entre eux avec une référence de calculation unique. tique et en toute connaissance de cause, afin de conserver notre liberté thérapeutique intacte. C'est ainsi que la FMH va poursuivre son accompagnement actif des développements tarifaires hospitaliers; elle devra toujours le faire d'une seule voix, condition indispensable au maintien de notre crédibilité dans ce domaine.

Dr Pierre-François Cuénoud, membre du Comité central, responsable du Domaine tarifs et économie de la santé pour les médecins hospitaliers 